\title{
Theoretical Chemistry and Electrochemistry
}

\section{Foreword}

This issue of Journal of Chemical Sciences contains papers in two major areasTheoretical Chemistry and Electrochemistry. These papers have been contributed by friends and former collaborators of Professor S K Rangarajan (SKR), who passed away on 29 April 2008. SKR has contributed very significantly to these areas and thereby promoted the growth of these disciplines in India. The contributions of SKR extended over a period of more than 50 years, during which he worked at the Central Electrochemical Research Institute (CECRI), Karaikudi, the National Aerospace Laboratories (NAL), Bangalore, the Indian Institute of Science (IISc), Bangalore and the Institute of Mathematical Sciences (IMSc), Chennai.

During the period at CECRI, SKR made significant contributions to (i) theory of Faradaic rectification; (ii) effect of diffuse double layer on electrode kinetics; (iii) accelerated Tafel plots for measuring rates of corrosion; (iv) estimation of the activity coefficients for mixed electrolytes, beyond the Debye-Huckel theory and (v) novel identities for a class of special functions of mathematical physics. As a Homi Bhabha Fellow at NAL during 1970-1975, Rangarajan embarked upon developing a Systems Analysis approach to all classes of electrochemical experiments (transient and steady state) with diverse input functions (linear and non-linear potential perturbations of diverse genre). This formalism encompasses almost all the individual experimental behaviour arising from chronoamperometry, chronopotentiometry, cyclic voltammetry, impedance spectroscopy, etc. for various electron transfer processes, coupled with mass transfer effects. In addition, he developed a comprehensive theory of electrical double layer employing statistical mechanical models and functional analysis, which occupies a central place in interfacial electrochemistry.

Subsequent to his stay at NAL, SKR served as a Professor at the Department of Inorganic and Physical Chemistry, Indian Institute of Science, Bangalore till 1990. His significant accomplishments during this period include the stochastic models of electrochemical systems, mechanism of electrochemical crystallization, adsorption isotherms at electrode surfaces, many-body theory of chemisorption and electron transfer, models for surface roughness, nucleation and growth phenomena and theory of charge transfer processes at semiconductor/electrolyte interfaces. His objective in all these investigations has been to provide a correct description of existing experimental observations and predict new experimental phenomena under suitable 'control variables'. On lien from IISc, he became the Director of CECRI during 1990-1993 where he directed a large group of researchers in applied electrochemistry and utilized his expertise in the analysis of corrosion phenomena, underpotential deposition of metals, current distribution in bipolar cells, chemically modified electrodes and related areas. After superannuation, SKR joined the IMSc, Chennai as a Senior Professor and investigated theory of divergent series and properties of Riemann Zeta functions. After completion of his term at IMSc, he joined the Raman Research Institute, Bangalore as an Emeritus Professor and was frequent visitor to his parent department (Inorganic and Physical Chemistry), at the IISc till his untimely death.

SKR occupied various positions in diverse Institutions and provided his complete wisdom and expertise to them, mentoring a generation of students and colleagues, irrespective of their formal background. He was always accessible for scientific and scholastic pursuits in mathematics, physics or chemistry. His intuitive knowledge coupled with rigorous mathematical training enabled him to comprehend scientific questions in a manner, 
virtually impossible for many. He was revered and held in high esteem by his colleagues and students, all over the world.

SKR has published around 200 papers in various Journals and wrote a review on electrical double layer in the Annual Specialist Periodical Reports brought out by the Chemical Society London. He received several honours and recognitions and among them, mention may be made of the Fellowship of the Indian Academy of Sciences, Bangalore, Indian National Science Academy, Third World Academy of Sciences, Palit Lectureship and Life time achievement award of the Chemical Research Society of India. He was the Member of the Editorial Board of Electrochimica Acta.

The papers presented in this issue cover a broad spectrum of topics in theoretical chemistry such as electronic structure calculations, quantum dynamics, magnetic properties, solvation dynamics and molecular dynamics. In electrochemistry, the papers discuss a variety of topics like electrochemical nanostructures, photoelectrochemistry, electron transfer, capacitors, etc. We hope that this issue of Journal of Chemical Sciences will be a fitting tribute to the scientific genius of SKR.

We wish to thank all the authors who have contributed to this issue. We also thank the Editor, Journal of Chemical Sciences and the Editorial Staff of the Journal for their support and cooperation.

25 September 2009

\author{
M V SANGARANARAYANAN \\ Department of Chemistry, \\ Indian Institute of Technology Madras, \\ Chennai 600036 \\ e-mail: sangara@iitm.ac.in
}

K L SEBASTIAN

Department of Inorganic and Physical Chemistry, Indian Institute of Science, Bangalore 560012 e-mail: kls@ipc.iisc.ernet.in

(Guest Editors) 